\title{
A research paper on the performance analysis of a Self-mode Locking high power linearly polarised fiber laser
}

\author{
Bishal Poudel, Om Nath Acharya \\ Department of Electrical and Electronics Engineering, Kathmandu University, Dhulikhel, Nepal
}

Email address:

poude1407@yahoo.com(B. Poudel), acharya.om@ku.edu.np(O. N. Acharya)

\section{To cite this article:}

Bishal Poudel, Om Nath Acharya. A Research Paper on the Performance Analysis of a Self-Mode Locking High Power Linearly Polarised Fiber Laser. American Journal of Optics and Photonics. Vol. 2, No. 4, 2014, pp. 45-53. doi: 10.11648/j.ajop.20140204.11

\begin{abstract}
In this research a design for high power linearly polarized all-fiber linear cavity lasers with self-mode-locking is presented, and a new theoretical model based on a Nonlinear Schrödinger Equation (NLSE) is developed and implemented on the MATLAB platform. For the design of cavity, Polarization Maintaining (PM) fibers for both the gain medium and the Fiber Bragg Gratings (FBGs) is implemented. The FBG pairs are used to select the lasing wavelength and polarization. The fiber lasers incorporate specially designed FBGs to achieve an extinction ratio larger than $23 \mathrm{~dB}$. The proposed configuration is based on Non-Linear Polarization (NPR) using PM Yb-doped active fiber and two matching FBGs to form the laser cavity. The combination of nonlinearity, gain, and birefringence in the cavity made the laser generate mode-locked pulses in the picosecond range and with high average output power. Experimental data and numerical simulations of the self-mode-locking fiber laser are presented. Main parameters affecting mode-locked pulses and its envelope are identified. The model proposed here explains the self-mode-locking mechanism and the source of the pulse envelope. In this model, it is proven that self-phase modulation (SPM) plays an essential role in pulse formation and shaping.
\end{abstract}

Keywords: Self-Mode-Locking (SML), Polarisation Maintaining (PM), Nonlinear Schrödinger Equation (NLSE), Fiber Bragg Gratings (FBGs), Optical Coupler (OC)

\section{Introduction}

Fiber lasers have extended their performance capabilities and substitute conventional laser technologies markets because of large number of benefits [1-3]. With the adoption of double-clad fibers and clad-pumping techniques, laser output can be obtained with high power and energy levels at either $\mathrm{CW}$ or pulsed regime. Fiber lasers are generally used in those applications which require high peak power and pulse energy. The two most well-established techniques to pulse fiber lasers are Qswitching and mode locking in either active or passive form $[4,5-7]$.

Mode-Locking of a laser produces ultra-short pulses on the order of picoseconds or femto-seconds. This technique induces a fixed phase relationship between the longitudinal modes of the laser's cavity. Interference between these modes causes the laser light to produce a train of pulses.
Locking of mode phases enables a periodic variation in the laser output which is stable over time, and with periodicity given by the round trip time of the cavity [7]. Such a laser is said to be mode-locked or phase-locked. In a real modelocked laser, the actual pulse width depends on other factors, such as the pulse shape and the overall dispersion of the cavity. Fiber lasers can be mode-locked either in active or passive way. Active methods typically use an external signal to modulate the cavity loss and consequently require the use of bulky modulators and drivers. Passive mode-locking depends on incorporating a SA in the laser cavity. A saturable absorber is an optical component with a certain optical loss, which is reduced at high optical intensities [4].

\subsection{Passively Mode-Locked Fiber Lasers}

Literature review shows that the most popular Saturable Absorbers in the $1 \mu \mathrm{m}$ band are Semiconductor Saturable 
Absorber Mirrors (SESAMs) [8-14], and Nonlinear Polarization Rotation (NPR) [15-19]. Other modulators such as Single-Wall Carbon Nanotubes (SWCNT) [20-25] and NOLM [26] are also reported. The fastest mode-locked pulse with pulse width 128fs [26] was achieved by combining nonlinear NPR and a nonlinear amplifying loop mirror.

\subsection{Self-Pulsed Fiber Lasers}

Two kinds of self-pulsing are mainly observed; sustained self-pulsing (SSP) and self-mode locking (SML) [14]. SSP is the emission of laser pulses at a repetition rate associated with relaxation oscillations and observed at specific pumping ranges. Most often, SSP has irregular repetition periods with pulses in microsecond and nanosecond ranges. Alternatively, more interests are into SML with pulse widths in the range of nanosecond down to picoseconds. In SML, the laser signal modulation period corresponds to the cavity round-trip time. Various mechanisms may cause self-pulsing in fiber lasers [28] including ion-pairing [29], re-absorption of laser photons in un-pumped part of fiber [30], scattering processes such as SBS or SRS [31] and Kerr effect [32]. In References [29-32], self pulsing were investigated only experimentally without numerical verification. Recently, Bedyakova et al. studied in [33] the pulsing dynamics of $\mathrm{Yb}$-doped linear fiber laser. The model is based on NLSE and includes both nonlinear and dispersion effects. Numerical simulations show good agreement for power and spectrum measurements. Yet, the model addresses only a narrow time scale of the self-pulsed output.

\section{System Model}

\subsection{Purposed Configuration of Fiber Laser}

The schematic of the proposed Self Mode-Locked (SML) all-PM fiber laser, with all fiber connections fusion spliced, is shown in Figure 2.1. The fiber laser was pumped by a fiber-coupled laser diode at $975 \mathrm{~nm}$ wavelength. Laser diodes of $10 \mathrm{~W}$ and $25 \mathrm{~W}$ were used and which have a multi-mode output fiber. In some configurations, the laser diode was connected to a low pass filter to pass pump wavelength and attenuate longer back-reflected wavelengths that can burn the laser diode. Though the filter introduced around $10 \%$ loss to the input pump power, it provided protection to the laser diode at high power levels.
The fiber laser cavity consists of an active fiber spliced between a FBG pair. Both active fiber and FBG fiber are of PM type. The FBG pair is formed of two gratings; one end of the highly reflective FBG is spliced to the laser diode or the filter and the other end is co-spliced (where both PM fibers axes are parallel to each other) to the active fiber. The other FBG is used as the cavity output coupler with reflectivity value ranges from $7 \%$ up to $82 \%$. In order to select polarization, the output coupler grating is crossspliced (where the slow axes of both PM fibers are perpendicular to each other) to the active fiber. Most of the laser modules have a PM APC-pigtail co-spliced to the output coupler FBG for reducing back reflection. In most cases, the pump diodes are operated continuously.

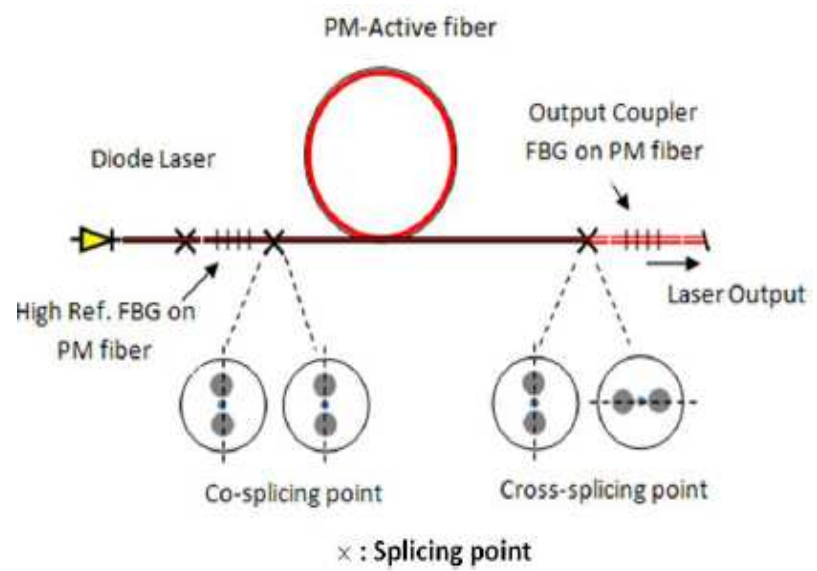

Figure 2.1. A schematic diagram of the linearly polarized mode-locked high-power fiber laser

\subsection{Active Fibers}

Two types of double-clad PM type active fibers are used in this research, Ytterbium (Yb3+) and Erbium-Ytterbium co-doped $(\mathrm{Er} 3+/ \mathrm{Yb} 3+)$. Ytterbium ions are one of the most versatile ions to be doped in silica-based fibers. It offers several advantages of wide absorption band from $850 \mathrm{~nm}$ to $1070 \mathrm{~nm}$. It can be also pumped with wide selection of solid-state laser diodes $(850 \mathrm{~nm}, 980 \mathrm{~nm}, 1047 \mathrm{~nm}$ and $1064 \mathrm{~nm}$ ). In addition, ytterbium co-doped fibers have impressive emission spectra in the range from 970 to 1200 $\mathrm{nm}$ [5]. In the research, all of our Yb-doped active fibers are pumped with $975 \mathrm{~nm}$ laser diodes with an emission in the $1 \mu \mathrm{m}$ band.

Table 2.1. Specifications of Er3+/Yb3+-doped and $Y b 3+$ active fibers used in the research.

\begin{tabular}{lll}
\hline Parameter & Er3+/Yb3+-doped active fiber (6/125) & Yb3+ doped active fiber (10/125) \\
\hline Fiber part number & Nufern PM-EYDF-6/125-HE & Nufern PLMA-YDF-10/125 \\
Fiber type & PM (PANDA), Double-clad & PM (PANDA),Double-clad \\
Core diameter/Cladding diameter & $6 \mu \mathrm{m} / 125 \mu \mathrm{m}$ & $10 \mu \mathrm{m} / 125 \mu \mathrm{m}$ \\
Mode field diameter & $6.7 \mu \mathrm{m}$ & $11 \mu \mathrm{m}$ \\
Core N.A. & 0.18 & 0.075 \\
Birefringence & 0.00034 & 0.0003 \\
Cladding absorption @ $975 \mathrm{~nm}$ & $0.89 \mathrm{~dB} / \mathrm{m}$ & $5 \mathrm{~dB} / \mathrm{m}$ \\
\hline
\end{tabular}


One of the most important parameters for active fiber is the absorption per meter at the pump wavelength. Most of the active fiber data sheets list the absorption per meter at $915 \mathrm{~nm}$ wavelength. The ratio between the absorption at $975 \mathrm{~nm}$ and $915 \mathrm{~nm}$ could be estimated from the absorption spectra. The absorption coefficient at $975 \mathrm{~nm}$ is about three times the one at $915 \mathrm{~nm}$ for $\mathrm{Yb} 3+$ while for $\mathrm{Er} 3+/ \mathrm{Yb} 3+$ co-doped fiber, the ratio varies from 5-7 [39-40]. This coefficient is important to estimate the length of the active fiber needed to build an efficient cavity. A minimum of 10$12 \mathrm{~dB}$ absorption of a gain fiber is required to build an efficient laser. The main specifications for active fibers used in the research are shown in both Table.2.1.

Fiber Bragg Gratings (FBGs) inscribed on a PM fiber are used in this research to build fiber laser cavities with a linearly polarized output. When the PM fiber is exposed to UV- light for grating inscription, the resulting index modulation gives rise to two spectrally separated Bragg reflective peaks because of fiber's birefringence [41]. In order to provide both polarization and wavelength selection, the fast axes wavelength of the high reflection (HR) FBG is matched to the slow axis wavelength of the output coupler FBG. The maximum mismatch allowed between both wavelengths is around $30-40 \mathrm{pm}$. The high isolation between the two FBG axes is a key factor to have a laser output with high extinction ratio. In order to maintain high isolation, the FBGs $3 \mathrm{~dB}$ bandwidth should not exceed the Bragg wavelength separation of the PM fiber. Writing FBG with a broader bandwidth will lower the isolation and may lead to dual polarization operation. On the other hand a too narrow bandwidth will make the wavelength matching difficult. In terms of splicing, high reflection FBG is cospliced (where both PM fibers are spliced with both their slow axes are parallel to each other) to one end of the active fiber. The output coupler FBG is cross-spliced (where both PM fibers are spliced with their slow axes are perpendicular to each other) to the other end of the active fiber.

\subsection{Fiber Laser Assembly}

Fiber laser assembly consists of two procedures, fiber splicing and fiber routing. Different types of splicing points and recoating types involved in assembly will be discussed in this section. Two types of splices were implemented as shown in the laser schematic in Figure2.1. The first is between multimode fiber and PM fiber (The point between the laser diode output or the bandpass filter (BPF) and the HR FBG). This type of splicing can be performed with an Ericson FSU-975 arc fusion splicer. The second type is between two PM fibers using a Fujikura FSM-20PMII arc fusion splicer. It has a program to splice two PM fibers at any desired angle. In the proposed laser module, the PM fiber splicing has two orientations; co-splicing or cross splicing. On the other side, cross-splicing of two PM fibers, is splicing the two PM fibers with their slow axes are perpendicular to each other.

\section{Numerical Modelling of All-PM Mode-Locked Fiber Laser}

\subsection{Nonlinear Pulse Propagation in Fiber}

The performance of picoseconds and femtosecond modelocked fiber lasers particularly in terms of pulse energy, peak power and pulse quality is controlled by the nonlinearities, dispersion and the gain of the fiber. In order to analyze the pulse formation and stabilization inside optical fiber, it is useful to introduce two length scales, known as the dispersion length LD and the nonlinear length LNL [38].

Based on the definition of dispersion and nonlinear length, two regimes for fiber and pulse interaction can be explained as:

a) Dispersion dominant regime: In this regime, the fiber length $\mathrm{L}<<\mathrm{LNL}$ but $\mathrm{L} \sim \mathrm{LD}$. The pulse evolution is governed by group velocity dispersion (GVD) and the nonlinear effects play a minor role.

b) Non-linearity regime: In this regime the fiber length $\mathrm{L}<<\mathrm{LD}$ but $\mathrm{L} \sim \mathrm{LNL}$. The dispersion has negligible effects compared to nonlinear term.

When the fiber length $\mathrm{L}$ is longer or comparable to both LD and LNL, dispersion and nonlinearity both act together as the pulse propagates along the fiber. The interplay of the dispersion and nonlinear effects can lead to a different behaviour [42] compared with the expected from dispersion or nonlinear alone [42, 43]. Nonlinear effects in optical fibers are particularly important when considering the propagation of short pulses (from $10 \mathrm{~ns}$ to $10 \mathrm{fs}$ ). The intensity-dependent refractive index of silica gives rise to three effects: Self-Phase Modulation (SPM) [44], CrossPhase Modulation (XPM) [42, 44] and Four-Wave Mixing (FWM). More insight into some of the fiber non-linearities such as SPM, XPM and NPR will be discussed in the following sections.

The Nonlinear Polarization Rotation (NPR) can be treated as a kind of artificial saturable absorption to achieve the passive mode locking in a fiber laser [45]. The evolution of the two polarization components will be tracked by a column matrix for the electric field and a $2 * 2$ matrix for the resonator elements. The electric field $2 * 1$ field vector is given by [45].

\subsection{Numerical Modelling}

Nonlinear Schrödinger Equation (NLSE) comes in a variety of forms based on the system being studied. Its generalized time domain form can be represented in [38]. The derivation of NLSE starting from Maxwell equation is illustrated in details in [35]. The time-domain Coupled NLSE (CNLSE) for high birefringence fiber is given in [46].

Split Step Fourier Method is one of the powerful 
methods to solve NLSE is known as split-step Fourier method (SSFM) which is the technique of choice for solving NLSE due to its easy implementation and speed compared to other methods especially time-domain finitedifference method [47, 48]. It assumes that mathematical terms due dispersion and nonlinearity can act separately and independently over short piece of fiber. The SSFM is an iterative process that determines the field solution for spatial steps of $h$. This performed for each step for the entire length of the fiber. The procedure during one step is illustrated in Figure 3.1. The fiber of length $\mathrm{L}$ is divided into $\mathrm{sL}=\mathrm{L} / \mathrm{h}$ steps of length $\mathrm{h}$ [48]. The field propagation $\mathrm{A}$ $(j h, t)$ per spatial step $h$ at the step $j h(j=1,2, s L)$ for the entire fiber length can be represented by the following relation:

$$
A(j h, t)=\mathcal{F}^{-1}\{\exp (h \hat{D}(i \omega)) F\{\exp (h \widehat{N}) A((j-1) h, t)\}\}
$$

where $A((j-1) h, t)$ is the field solution from the previous step. The operation of nonlinear operator occurs in the middle of the step. It is the first operator to act on $\mathrm{A}(\mathrm{jh}, \mathrm{t})$. The effect of dispersion is determined in the frequency domain. The field solution is Fourier transformed, operated on by and then back transformed to give solution $A(j h, t)$ at step $\mathrm{jh}$. The field solution contains both intensity and phase information.

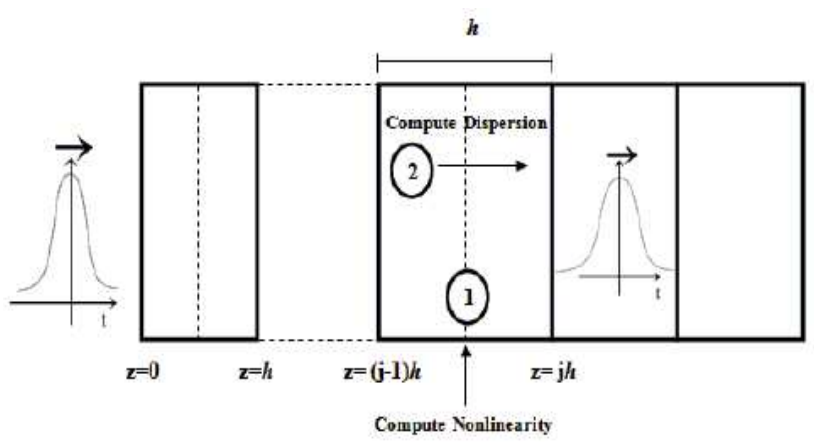

Figure 3.1. The SSFM for one iteration of step $h$ starting at $z=(j-1) h$. The initial pulse $A(0, t)$ enters the medium of length $L$. The length is divided into $s L=L / h$ steps of length $h$.

\subsection{Simulation Model}

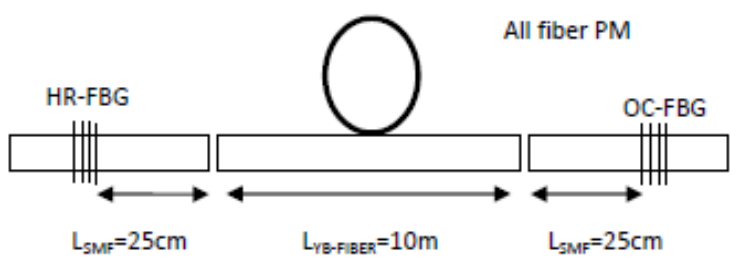

(a)

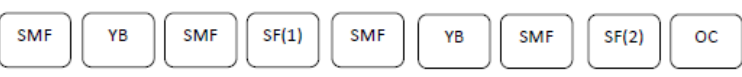

(b)

Figure 3.2. (a) Fiber laser schematic (b) Simulation model schematic; where $S F(1)$ is the HR-FBG and $S F(2)$ is the $O C-F B G$.
The fiber laser cavity (Figure 3.2(a) is divided into the sections and modeled as shown in Figure 3.2(b). The proposed simulation model considers the following:

1. NLSE is used to simulate the laser cavity

2. Two sections of single mode fiber (SMF) are included in the cavity modelling (on which FBG is written).

3. Any FBG in the laser represents Spectral filtering component.

4. The OC-FBG acts also as an output coupler.

Following the approach in $[49,50]$, the operator, C, describing the round trip for the linear cavity can be represented as follows:

$$
C=F G e^{i \varphi} F R_{H R} F G e^{i \varphi} F R_{O C}
$$

Where, $F$ and $G$ represent the propagation through the SMF and the gain fiber. $\varphi$ is a cavity related phase shift. RHR and ROC are the reflectivity of the HR and OC FBGs respectively. Each component model will be explained as follows:

\section{a) Fiber Sections}

In the simulation, the pulse propagation through SMF and gain fiber represented by $F$ and G are solved by NLSE [38] using a split-step Fourier method. The gain saturation model is given in Eq. (3.22), where g0 is the small signal gain coefficient (related to dopant concentration), $\mathrm{E}$ is the instantaneous pulse energy and Esat is the gain saturation energy which is pump-power dependent. For the non-gain single mode fiber, saturated gain factor $\mathrm{g}$ is set to zero to eliminate the influence of the gain effect.

$$
g=\frac{g_{0}}{1+\frac{E}{E_{\text {sat }}}} \text { and } T_{2}=\frac{2}{\Delta \omega_{g}}
$$

If two polarizations are included in the cavity simulation, NLSE is replaced by CNLSE. The model assumes equal gain for the two polarizations and the high Birefringence fiber Jones matrix [51] is given as:

$$
T=M_{H i-B i}=\left(\begin{array}{cc}
e^{-i(2 \pi, \Delta n \cdot L) / \lambda} & 0 \\
0 & e^{i(2 \pi, \Delta n \cdot L) / \lambda}
\end{array}\right)
$$

Where $\mathrm{L}$ is the length of the fiber, $\Delta \mathrm{n}$ is the fiber birefringence and $\lambda$ is the operating wavelength. Other components of the round-trip model like gain, saturable absorption, mirrors, etc., usually do not distinguish between the two polarization components. The output signal after gain fiber [52, 53] will be:

$$
\left(\begin{array}{l}
E^{\text {out }}{ }_{x} \\
E^{\text {out }}{ }_{y}
\end{array}\right)=\sqrt{ } G *\left(\begin{array}{cc}
e^{-i(2 \pi \cdot \Delta n \cdot L) / \lambda} & 0 \\
0 & e^{i(2 \pi \cdot \Delta n, L) / \lambda}
\end{array}\right) *\left(\begin{array}{l}
E^{\text {in }}{ }^{x} \\
E^{\text {in }}{ }_{y}
\end{array}\right)
$$

Regarding the SSFM, the nonlinear operator should be changed to: 


$$
\begin{aligned}
& \widehat{N_{x}}=i \gamma\left(\left|A_{x}\right|^{2}+2 / 3\left|A_{y}\right|^{2}\right) \\
& \widehat{N_{y}}=i \gamma\left(\left|A_{y}\right|^{2}+2 / 3\left|A_{x}\right|^{2}\right)
\end{aligned}
$$

\section{b) Cavity phase shift component}

The cavity phase shift, $\varphi$ induced from the gain fiber section and represents the polarization rotation, is expressed as shown in Eq. (3.26).

$$
\varphi=\varphi_{L}+\varphi_{N L}=\frac{-2 \pi \Delta n L_{y b}}{\lambda_{0}}+\gamma_{Y b} L_{Y b}|A|^{2}
$$

where $\mathrm{n}$ is the birefringence of the PM fiber. ${ }^{L_{Y b}}$ and $\gamma_{Y b}$ are the length and nonlinear coefficient of the gain fiber respectively. $\lambda_{0}$ is the center wavelength. The first term in Eq. (3.26) represents the linear birefringence of the PM fiber $[54,55]$ while the second is a nonlinear phase $\mathrm{m}$ shift proportional to the intra-cavity field power. This term in the model is part of the pulse phase shift evolution and rotation that plays a major role in the performance of the SML laser. If two $m$ polarizations are included in the cavity simulation, NLSE should be replaced by CNLSE. At such as case, a new cavity phase shift for each polarization will be introduced as:

$$
\begin{gathered}
\varphi_{x}=\frac{-2 \pi \Delta n L_{y b}}{\lambda_{0}}+\gamma_{Y b} L_{Y b}\left(\left|A_{x}\right|^{2}+2 / 3\left|A_{y}\right|^{2}\right) \\
\varphi_{y}=\frac{2 \pi \Delta n L_{y b}}{\lambda_{0}}+\gamma_{Y b} L_{Y b}\left(\left|A_{y}\right|^{2}+2 / 3\left|A_{x}\right|^{2}\right)
\end{gathered}
$$

c) FBG (Spectral filtering components and Output coupler)
Both HR-FBG (RHR) and OC-FBG (ROC), their reflectivity spectra are modeled with a Gaussian function with a bandwidth of $0.2 \mathrm{~nm}$ and center wavelength $\lambda_{\mathrm{o}}$ of $1064 \mathrm{~nm}$. The output is taken at the 50\% OC-FBG. The filter function is represented in frequency domain [52] as shown in Eq. (3.28). That is the reason to apply FFT for the input signal before applying to filter section. At the filter output, IFFT operation is applied to the output signal.

$$
S F(f)=\exp \left(-2 \ln 2 \frac{f^{2}}{\Delta f^{2}}\right)
$$

where SF (f) is the filter transfer function in frequency domain and $\Delta \mathrm{f}$ is the filter's $3 \mathrm{~dB}$ bandwidth.

For the two polarization simulations, PM FBG will be modeled with a reflectivity matrix with two wavelengths $[67,68]$ and spectral filtering component as

$$
\left(\begin{array}{c}
A^{\text {out }}{ }^{x} \\
A^{\text {out }}{ }_{y}
\end{array}\right)=S F *\left(\begin{array}{cc}
r_{1} & 0 \\
0 & r_{2}
\end{array}\right) *\left(\begin{array}{c}
A^{\text {in }}{ }_{x} \\
A^{\text {in }}{ }_{y}
\end{array}\right)
$$

$\mathrm{r} 1$ and $\mathrm{r} 2$ is the reflectivity of both PM FBG axes.

d) Initialization Pulse

The simulation is initialized by small amplitude Gaussian pulse of FWHM of 1ns. For the two polarization

\begin{tabular}{|c|c|c|}
\hline Gain fiber & SM fiber & FBGs \\
\hline Fiber Length $(\mathrm{L})=10 \mathrm{~m}$ & Fiber Length $(\mathrm{L})=0.25 \mathrm{~m}$ & HR-FBG reflectivity $(\mathrm{RHR})=0.99$ \\
\hline $2 \mathrm{nd}$ order dispersion $(\beta 2)=0.024 \mathrm{ps} 2 / \mathrm{m}$ & 2 nd order dispersion $(\beta 2)=0.024 \mathrm{ps} 2 / \mathrm{m}$ & OC-FBG reflectivity $(\mathrm{ROC})=0.5$ \\
\hline Nonlinear coefficient $(\gamma)=0.0016 \mathrm{~W}-1 \mathrm{~m}-1$ & Nonlinear coefficient $(\gamma)=0.0016 \mathrm{~W}-1 \mathrm{~m}-1$ & $\mathrm{FBG} 3 \mathrm{~dB}$ BW $(\Delta \lambda 3 \mathrm{~dB})=0.2 \mathrm{~nm}$ \\
\hline Small signal gain $(\mathrm{go})=0.1 \mathrm{~m}-1$ & & \\
\hline Saturation energy (Esat) $=2 \mathrm{~nJ}$ (Pump parameter) & Small signal gain $(\mathrm{go})=0$ & Center wavelength $(\lambda \mathrm{o})=1064 \mathrm{~nm}$ \\
\hline Gain bandwidth $(\Delta \omega \mathrm{g})=45 \mathrm{~nm}$ & & \\
\hline Birefringence $(\Delta n)=3 \times 10-4$ & Birefringence $(\Delta n)=3 \times 10-4$ & - \\
\hline
\end{tabular}
simulation, two Gaussian pulses will be used to represent each polarization as shown in Eq. (3.30).

$$
\left(\begin{array}{c}
E^{i n i}{ }_{x} \\
E^{i n i}{ }_{y}
\end{array}\right)=\left(\begin{array}{c}
E_{x} \exp \left(-\frac{t}{T o}\right)^{2} \\
E_{y} \exp \left(-\frac{t}{T o}\right)^{2}
\end{array}\right)
$$

\subsection{Simulation Parameters \& Results}

Table 3.1. Simulation parameters for NLSE-based model.

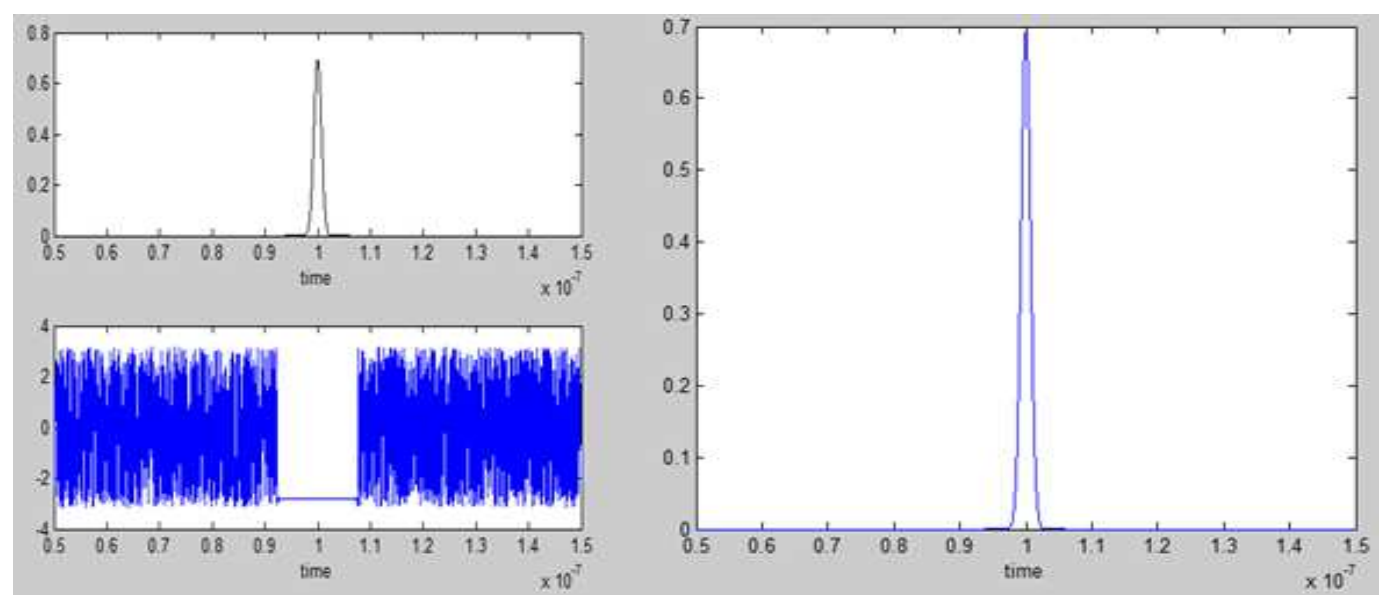



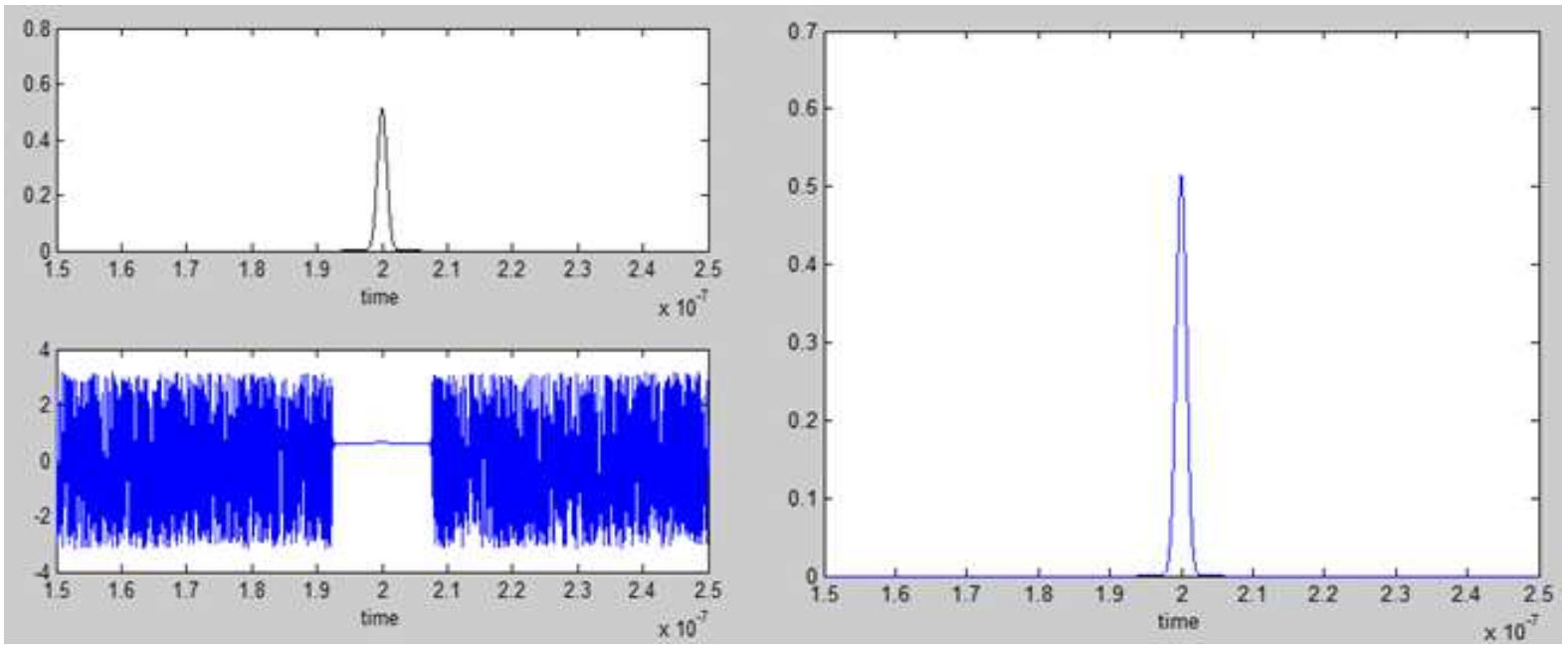

b
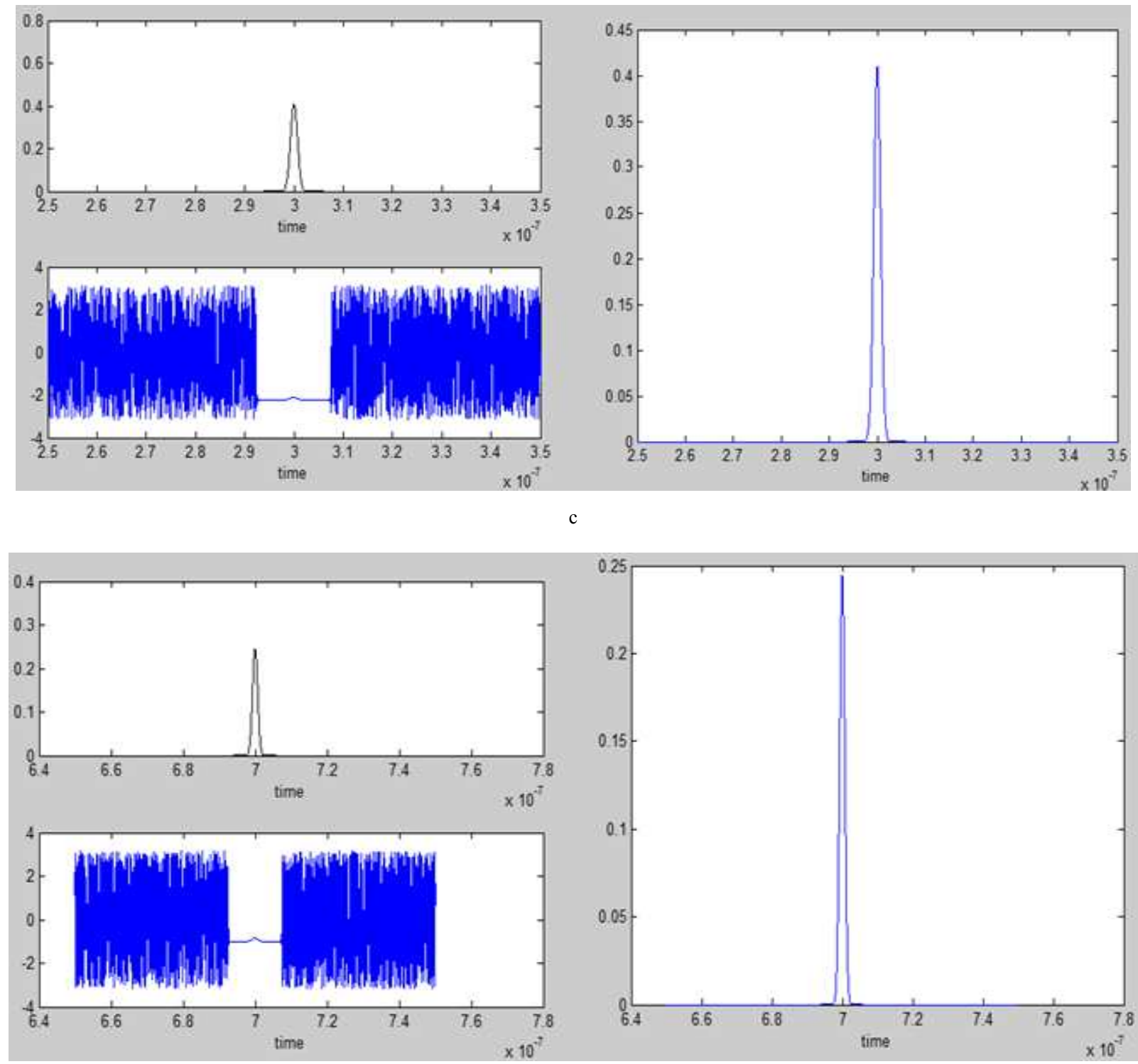


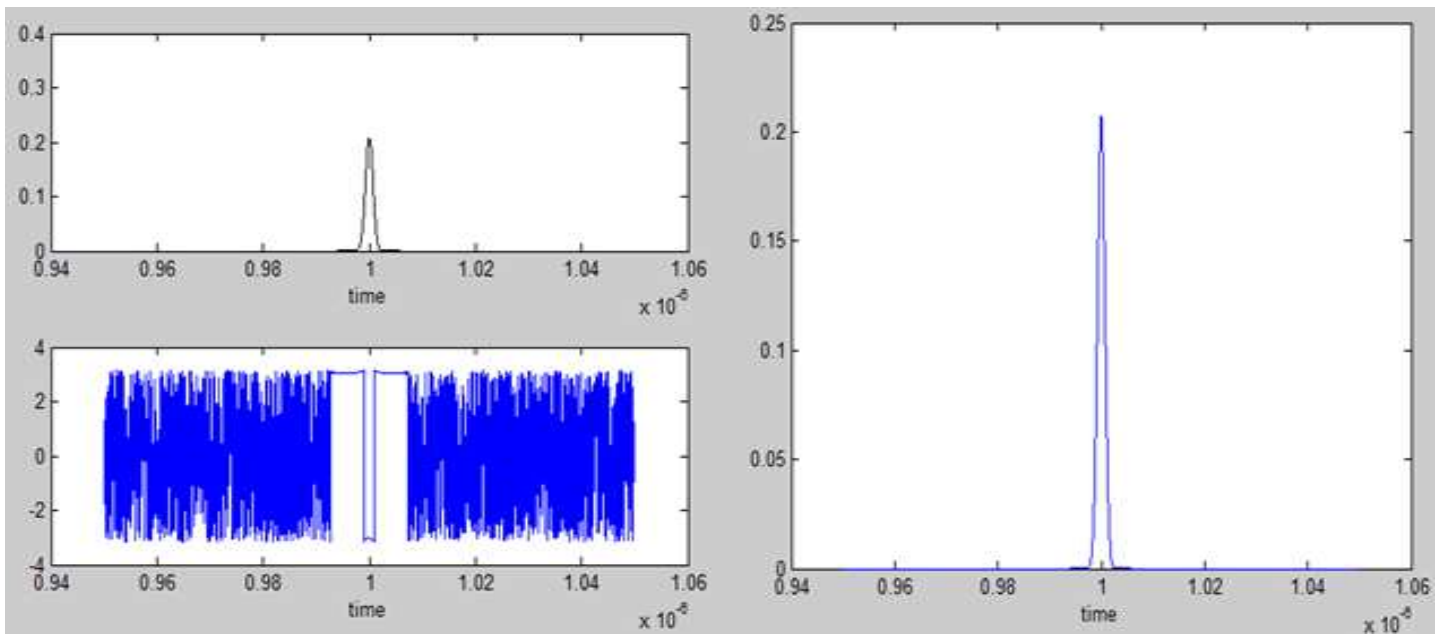

Figure 3.3. (a-e) one shot mode locked pulses

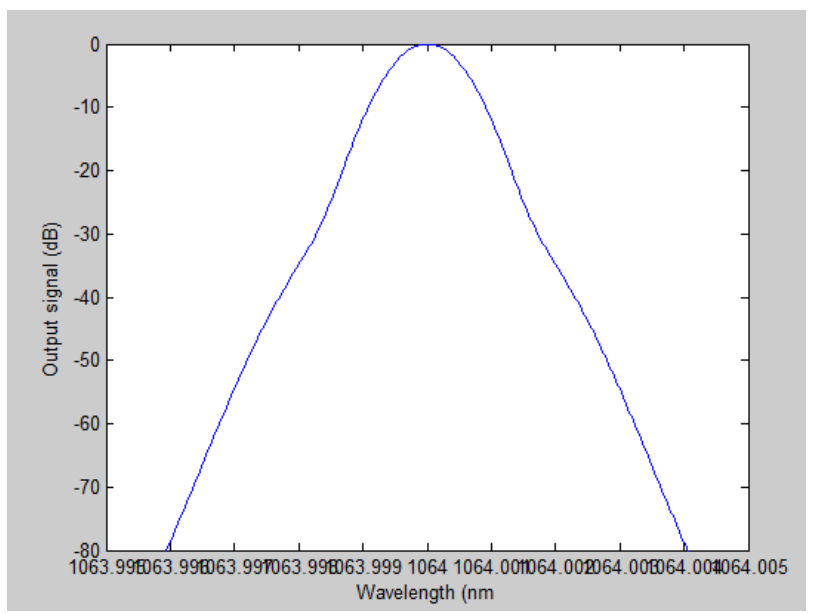

Figure 3.4. Simulated output pulses linewidth with mode locking

The numerical simulation starts with a 1 ns Gaussian pulse as an initial condition and we examine the output after 10000 round trips. In one round-trip, the propagation of the optical pulses is solved in every section of the laser. After one round-trip through the laser cavity, the resulted pulse from simulation was used as the input for the next round-trip calculation. The outcome results of the proposed model are studied at different conditions. The model is first tested without adding the phase shift parameter to verify its role in pulse formation. The simulation output without and with this term is shown in Figure 3.3(a) to (e) and overall linewidth after mode-locking is shown in figure 3.4.

The figure 4.8 shows the line-width ranging from $1063.995 \mathrm{~nm}$ to $1064.005 \mathrm{~nm}$. This shows a very very precise line-width at $1064 \mathrm{~nm}$ of $0.01 \mathrm{~nm}$ wide. In this research when the iteration time is made 4000 times the pulse width becomes $0.01 \mathrm{~nm}$.

\section{Conclusions}

The mode-locking mechanism for the proposed laser can be explained using NPR. The laser is designed such that
PM-FBG pair selects one polarization which is amplified by the cavity. However, a small component of the other orthogonal polarization can also evolve inside the cavity. The walk-off between the two orthogonal polarizations accumulates while propagating along the cavity. Thus the propagating light will be elliptically polarized and its rotation becomes intensity dependent. When the elliptically polarized light reaches the OC-FBG, the light component aligned with fast axis will only pass it. Thus the laser output is linearly polarized. The PM-FBG pair in the laser cavity acts as a polarizer through which intensitydependent rotation is transformed into intensity dependent transmission. This serves as a fast artificial SA and generates mode-locked output pulses.

\section{Acknowledgements}

I would like to express my gratitude to my supervisor lecturer om nath acharya for his unreserved support and guidelines.

\section{References}

[1] M. E. Fermann and I. Hartl, "Ultrafast fiber laser technology," IEEE Journal of Selected Topics in Quantum Electronics, vol. 15, pp. 191-206, 01, 2009.

[2] D. D. Hudson, "Mode-locked fiber lasers: Development and application," 2009.

[3] Jeff Hecht, "Fiber lasers: The state of the art," Laser Focus World, vol. 48, pp. 57, 2012.

[4] P. Rüdiger, Encyclopedia of Laser Physics and Technology, 1ed, Wiely-VCH, 2008.

[5] M. J. F. Digonnet, Rare-earth-doped fiber lasers and amplifiers, 2d ed, Marcel Dekker, vol. 25, 2001.

[6] O. Svelto and D. C. Hanna, Principles of Lasers, Springer, 1998. 
[7] B. E. A. Saleh, M. C. Teich and B. R. Masters, "Fundamentals of Photonics, Second Edition," John Wiely \& Sons, 1991.

[8] B. C. Seung, H. Song, S. Gee and Y. K. Dug, "Self-starting passive mode-locked ytterbium fiber laser with variable pulse width," in Fiber Lasers VII: Technology, Systems, and Applications, 2010, pp. $75802 \mathrm{C}$ (11 pp.).

[9] G. H. Jang and T. H. Yoon, "Environmentally-Stable Allnormal-dispersion Picosecond Ybdoped Fiber Laser with an Achromatic Quarter-wave-plate," Laser Physics, vol. 20, pp. 1463-8,2010.

[10] F. Q. Lian, Z. W. Fan, X. F. Wang, Y. T. Huang, K. Huang, Y. F. Ma, G. Niu, X. H. Li and J. Yu, "Ytterbium doped allfiber-path all-normal dispersion mode-locked laser based on semiconductor saturable mirror," Laser Physics, vol. 21, pp. 1103-7, 06, 2011.

[11] X. Tian, M. Tang, X. Cheng, P. P. Shum, Y. Gong and C. Lin, "High-energy wavebreaking-free pulse from all-fiber mode-locked laser system," Optics Express, vol. 17, pp. 7222-7, 2009.

[12] R. Song, H. Chen, S. Chen, J. Hou and Q. Lu, "A SESAM passively mode-locked fiber laser with a long cavity including a band pass filter," Journal of Optics, vol. 13, 2011.

[13] J. Liu, J. Xu and P. Wang, "High Repetition-Rate Narrow Bandwidth SESAM Mode-Locked Yb-Doped Fiber Lasers," IEEE Photonics Technology Letters, vol. 24, pp. 539541,2012 .

[14] Oliver Prochnow, Axel Ruehl, Michael Schultz, Dieter Wandt and Dietmar Kracht, "Allfiber similariton laser at 1 $\mu \mathrm{m}$ without dispersion compensation," Optics Express, vol. 15, pp.6889-6893, 2007.

[15] K. Ozgören and F. O. Ilday, "All-fiber all-normal dispersion laser with a fiber-based Lyot filter," Opt. Lett., vol. 35, pp. 1296,2010

[16] M. Schultz, H. Karow, O. Prochnow, D. Wandt, U. Morgner and D. Kracht, "All-fiber ytterbium femtosecond laser without dispersion compensation," Optics Express, vol. 16, pp.19562-19567, 2008.

[17] J. Fe ete, A. serteg and . Szip ocs, "All-fiber, all-normal dispersion ytterbium ring oscillator," Laser Physics Letters, vol. 6, pp. 49-53, 2009.

[18] C. K. Nielsen and S. R. Keiding, "All-fiber mode-locked fiber laser," Opt. Lett., vol. 32,pp. 1474-1476, 2007.

[19] F. Wang, A.G. Rozhin, V. Scardaci, Z. Sun, F. Hennerich, H. White, W.I. Milne and A.C. Ferrari "Wideband-tuneable, nanotube mode-locked, fibre laser," Nature Nanotechnology, vol.3, pp. 738-742, 2008.

[20] R. Going, D. Popa, F. Torrisi, Z. Sun, T. Hasan, F. Wang and A. C. Ferrari, "500 fs wideband tunable fiber laser mode-locked by nanotubes," Physica E: Low-Dimensional Systems and Nanostructures, vol. 44, pp. 1078-1081, 2012.

[21] Y. Senoo, N. Nishizawa, Y. Sakakibara, K. Sumimura, E. Itoga, H. Kataura and K. Itoh, "Polarization-maintaining, high-energy, wavelength-tunable, Er-doped ultrashort pulse fiber laser using carbon-nanotube polyimide film," Optics Express, vol. 17, pp. 20233-20241, 2009.
[22] N. Nishizawa, Y. Nozaki, E. Itoga, H. Kataura and Y. Sakakibara, "Dispersion-managed, high-power, Er-doped ultrashort-pulse fiber laser using carbon-nanotube polyimide film," Optics Express, vol. 19, pp. 21874, 2011.

[23] Anonymous "A compact, high power, ultrafast laser modelocked by carbon nanotubes," Appl. Phys. Lett., vol. 95, pp. 253102-253102-3, 2009.

[24] Y. Hori, Z. Zhang and M. Nakazawa, "1070 NM passively mode-locked ytterbium-doped fiber soliton laser with SWNT/PMMA saturable absorber," in 2011, pp. 1-2.

[25] J. Peng, L. Zhan, Z. Gu, K. Qian, S. Luo and Q. Shen, "Direct generation of 128-fs Gaussian pulses from a compensation-free fiber laser using dual mode-locking mechanisms," Opt. Commun., vol. 285, pp. 731-733, 2012.

[26] W. Guan and J. R. Marciante, "Complete elimination of self-pulsations in dual-clad ytterbium-doped fiber lasers at all pumping levels," Opt. Lett., vol. 34, pp. 815-817, 2009.

[27] B. N. Upadhyaya, U. Chakravarty, A. Kuruvilla, S. M. Oak, M. R. Shenoy and K. Thyagarajan, "Self-pulsing characteristics of a high-power single transverse mode $\mathrm{Yb}$ doped CW fiber laser," Opt. Commun., vol. 283, pp. 2206$2213,2010$.

[28] A. V. Kir'yanov and Y. O. Barmenkov, "Self-Q-switched Ytterbium-doped all-fiber laser," Laser Physics Letters, vol. 3, pp. 498-502, 2006.

[29] Feng Xiao-xing, C. Bai, Chen Jia-lin, W. Li, Liang Li-ping and Lin Zun-qi, "Study of selfmode-locking in Q-switched and in all-fiber fabry-perot cavity ytterbium-doped fiber laser," inPassive Components and Fiber-Based Devices, 2005, pp. 88-95.

[30] B. N. Upadhyaya, A. Kuruvilla, U. Chakravarty, M. R. Shenoy, K. Thyagarajan and S. M. Oak, "Effect of laser linewidth and fiber length on self-pulsing dynamics and output stabilization of single-mode Yb-doped double-clad fiber laser," Appl. Opt., vol. 49, pp. 2316, 2010.

[31] Y. H. Tsang, T. A. King, D. Ko and J. Lee, "Output dynamics and stabilisation of a multimode double-clad Yb-doped silica fibre laser," Opt. Commun., vol. 259, pp. 236-241, 2006.

[32] A. E. Bednyakova, O. A. Gorbunov, M. O. Politko, S. I Kablukov, S. V. Smirnov, D. V. Churkin, M. P. Fedoruk and S. A. Babin, "Generation dynamics of the narrowband $\mathrm{Yb}$ doped fiber laser," Optics Express, vol. 21, pp. 8177, 2013.

[33] J. Jiang, J. Chang, S. Feng, L. Wei and Q. Mao, "Mid-IR multiwavelength difference frequency generation based on fiber lasers," Optics Express, vol. 18, pp. 4740, 2010.

[34] J. Chang, Q. Mao, S. Feng, X. Gao and C. Xu, "Widely tunable mid-IR differencefrequency generation based on fiber lasers," Opt. Lett., vol. 35, pp. 3486, 2010.

[35] J. H. Chang, Q. H. Mao, S. J. Feng, J. Jiang, X. L. Li, Y. Y. Tian, C. Q. Xu and W. Q. Liu,"Theoretical and experimental investigations of the Mid-IR DFG tuning property based on fiber laser fundamental lights," Applied Physics B, vol. 104, pp. 851-859, 2011.

[36] Li Xiu-li, Feng Su-juan and Mao Qing-he, "Development of fiber-type widely tunable DFG mid-infrared laser source," Chinese Journal of Quantum Electronics, vol. 29, pp. 298$302,05,2012$ 
[37] G. P. Agrawal, Nonlinear Fiber Optics, 3ed, Elsevier Science Academic Press, 2007, pp.11-13,63-66,97-104,213.

[38] G. C. Valley, "Modeling Cladding-Pumped Er/Yb Fiber Amplifiers," Optical Fiber Technology, vol. 7, pp. 21-44, 2001.

[39] D. T. Nguyen, D. T. Nguyen, A. Chavez-Pirson, A. ChavezPirson, Shibin Jiang, N. Peyghambarian and N. Peyghambarian, "A Novel Approach of Modeling CladdingPumped Highly Er-Yb Co-Doped Fiber Amplifiers," IEEE J. Quant. Electron., vol. 43, pp. 1018-1027, 2007.

[40] Deparis, O. Deparis, R. Kiyan, S. A. Vasiliev, V. O. I. Medvedkov, E. M. Dianov, O. Pottiez, P. Megret and M. Blondel, "Polarization-maintaining fiber Bragg gratings for wavelength selection in actively mode-locked Er-doped fiber lasers," IEEE Photonics Technology Letters, vol. 13, pp. 284-286, 2001

[41] R. G. Molla, "Nonlinear fiber optics for Bio-Sensing," M.Sc Thesis, University of Kansas, 2005.

[42] A. Yariv and P. Yeh, Photonics: Optical Electronics in Modern Communications, 6ed, Oxford University Press 2007, pp.251-273, 633-637.

[43] J. A. Buck and I. Jacobs, Fundamentals of Optical Fibers, Wiely Series in Pure and Applied Optics, 2ed, 2004, pp. 241-251

[44] J. Diels, Ultrashort Laser Pulse Phenomena, 2ed, Elsevier Science Academic Press, 2006, pp.63-66, 97-104, 317-318, 381-384

[45] C. K. Nielsen, "Mode-locked fiber lasers: Theoritical and Experimental Development," PhD. Thesis, University of Aarhus, Denmark, 2006.
[46] F. O. Ilday, Ultrashort pulse propagator v.2.2.2 user guide, Bilkent University, 2001.

[47] B. Washburn: www.phys.ksu.edu/personal/washburn/pdf/washburn_thesis chapter4.pdf

[48] J. Lægsgaard, "Control of fibre laser mode-locking by narrow-band Bragg gratings," Journal of Physics B: Atomic, Molecular and Optical Physics, vol. 41, pp. 095401, 2008.

[49] E. Paulucci, N. A. Russo, E. E. Sicre and R. Duchowicz, "Numerical and experimental comparison of an all-fiber APM laser with two-coupled linear cavities," Optics \& Laser Technology, vol. 48, pp. 495-502, 2013.

[50] J. Rogers, Polarization in Optical Fibers, The Artech House Applied Photonics Series, 2008 ,pp.94-98, 100-101.

[51] L. N. Binh and N. Q. Ngo, Ultra-Fast Fiber Lasers: Principles and Applications with MATLAB Models, CRC Press, 2010, pp.180-182.

[52] Y. Zhao, T. Song, D. Wu and Q. Wang, "Research on fiber optic temperature sensor using a novel high-birefringent fiber loop mirror with a reflection probe," Sensors \& Actuators: A.Physical, vol. 184, pp. 22, 2012.

[53] R. M. Silva, A. Layeghi, M. I. Zibaii, H. Latifi, J. L. Santos and O. Frazao, "Theoretical and Experimental Results of High-Birefringent Fiber Loop Mirror With an Output Port Probe," J. Lightwave Technol., vol. 30, pp. 1032-1036, 2012.

[54] F. Yang, Z. Q. Pan, Q. Ye, D. J. Chen, H. W. Cai and R. H. $\mathrm{Qu}$, "Effect of active fiber birefringence on polarization properties of DBR all-fiber laser," Laser Physics, vol. 22, pp. 778- 783, 2012. 